\title{
Yo soy lo que tú no eres: la alteridad en la definición de la identidad andaluza
}

\section{Am What You Are not: The Otherness in the Definition of the Andalusian Identity Eu sou 0 que você não é: a alteridade na definição da identidade andaluza}

\author{
Beatriz Macías-Gómez-Stern* \\ Universidad Pablo de Olavide, Sevilla, España. \\ Samuel Arias-Sánchez ${ }^{* *}$ \\ Universidad de Sevilla, Sevilla, España.
}

Doi: http://dx.doi.org/10.12804/revistas.urosario.edu.co/apl/a.5634

\section{Resumen}

En este artículo presentamos los resultados de una investigación empírica en la que exploramos el impacto de la emigración como experiencia de contraste con un "otro cultural" en la construcción de la identidad andaluza (Sur de España). Para ello hemos analizado el uso de comparaciones en narrativas identitarias (Bruner, 1991) de personas con distintas experiencias migratorias en grupos de discusión sobre la identidad andaluza. Se analizaron los datos discursivos usando un sistema de categorías de elaboración propia. Los resultados mostraron que las comparaciones como estrategia de construcción de la identidad en el debate eran diferencialmente empleadas por los tres grupos (emigrantes, no emigrantes y emigrantes retornados), siendo los emigrantes los que más recurrían a dicho recurso. También variaban la naturaleza y el grado de explicitud de los objetos y referentes de la comparación.
Como conclusión esgrimimos que la experiencia de emigración, como vivencia de contraste con un alter cultural, afecta el modo en el que las personas construimos nuestras identidades, siendo la comparación con este alter uno de los recursos más empleados para dicho proceso.

Palabras clave: identidad, alteridad, narrativas, migración.

\section{Abstract}

In this article, we present the results of an empirical research where we explore the impact of emigration, as an experience of contrast with a "cultural other" in the construction of the Andalusian identity (South of Spain). For this, we have analyzed the use of comparisons in identity narratives (Bruner, 1991) of people with different migratory experiences in discussion groups about the Andalusian identity. The discursive data was

* Beatriz Macías-Gómez-Stern, Facultad de Ciencias Sociales, Universidad Pablo de Olavide, Sevilla, España.

** Samuel Arias-Sánchez, Facultad de Psicología, Universidad de Sevilla, Sevilla, España.

La correspondencia relativa a este artículo debe ser enviada a Samuel Arias-Sánchez, Departamento de Psicología Experimental, Universidad de Sevilla, Avda. Camilo José Cela s/n, 41018, Sevilla, España. Correo electrónico: samuel@us.es

Cómo citar este artículo: Macías-Gómez-Stern, B. \& Arias-Sánchez, S. (2018). Yo soy lo que tú no eres: la alteridad en la definición de la identidad andaluza. Avances en Psicología Latinoamericana, 36(3), 493-509. DoI: http://dx.doi.org/10.12804/ revistas.urosario.edu.co/apl/a.5634 
analyzed using a system of self-developed categories. The results showed that the use of comparisons as a strategy for constructing identity in the debate was differentially used by the three groups (emigrants, non-migrants and returned migrants), with emigrants using this resource the most. The nature and degree of explicitness of the objects and references of comparison also varied. In conclusion, we argue that the experience of emigration, as an experience of contrast with a cultural other, affects the way in which we build our identities, being the comparison with this one of the most used resources for this process.

Keywords: Identity, otherness, narratives, migration.

\section{Resumo}

Neste artigo apresentamos os resultados de uma pesquisa empírica na que exploramos o impacto da emigração como experiência de contraste com um "outro cultural" na construção da identidade andaluza (Sul da Espanha). Para isso, temos analisado o uso de comparações em narrativas identitárias (Bruner, 1991) de pessoas com distintas experiências migratórias em grupos de discussão sobre a identidade andaluza. Se analisaram os dados discursivos usando um sistema de categorias de elaboração própria. Os resultados mostraram que as comparações como estratégia de construção da identidade no debate eram diferencialmente empregadas pelos três grupos (emigrantes, não emigrantes e emigrantes retornados), sendo os emigrantes os que mais recorriam a dito recurso. Também variavam a natureza e o grau de explicitação dos objetos e referentes da comparação. Como conclusão esgrimimos que a experiência de emigração, como vivência de contraste com um alter cultural, afeta o modo em eu as pessoas construímos nossas identidades, sendo a comparação com este alter um dos recursos mais empregados para dito processo. Palavras-chave: identidade, alteridade, narrativas, migração.

\section{Introducción}

Aunque se trate de un tema que ya podríamos denominar clásico en psicología, en las publicaciones más recientes de muy diversos ámbitos la pregunta por la identidad parece incesante y recurrente. Esteban-Guitart (2008), al analizar la importancia que se le da en la actualidad a la identidad, afirma que hoy es fruto de una doble desorientación en el plano individual o privado y en el colectivo y público, en la que la globalización juega un papel muy importante. A nivel individual, viene por la obligación de cuestionar las circunstancias sobre las cuales cada persona desarrolla su proyecto de vida en relación con la pareja o el trabajo, en un momento en el que los modelos tradicionales en los que el porvenir se dibujaba fácilmente están muy cuestionados. Por otro lado, los colectivos (nacionales, religiosos, étnicos, lingüísticos) reivindican su sitio y el derecho de "ser diferentes" en un mundo globalizado que cada vez nos hace a todos más iguales (Espinosa et al., 2017). El concepto de identidad es uno de los más complejos de investigar y conceptualizar desde la psicología.

Para dar un primer paso en la aclaración del concepto de identidad tal como se ha entendido en este trabajo, realizaremos, de un modo muy general y sin más pretensión que la de clarificar, un breve bosquejo de algunas teorías que sobre este constructo se han elaborado. Comenzaremos con las perspectivas que estudian las prácticas identitarias de los grupos humanos para ir al análisis del concepto de identidad cultural de las perspectivas más situadas. Con esto no pretendemos ni ser exhaustivos en el análisis de las múltiples perspectivas, ni mucho menos afirmar que unas definiciones de la identidad sean más complejas $o$ adecuadas que otras.

La identidad, o el yo, tanto individual como grupal, ha sido definida como una construcción 
dialógica que incluye una infinita polifonía de discursos resultado de nuestras interacciones con otras personas, opiniones y cosmovisiones. Nuestro yo es resultado de una dialéctica interna y externa de identificación, siendo esos discursos diversos los que le dan forma y sentido (Bakhtin, 1986). Esta concepción dialógica implica tanto un yo relacional en constante compromiso dialógico y ético con la sociedad, como nuevas estrategias de análisis (Clegg \& Salgado, 2011). Desde esta perspectiva y con una aproximación evolutiva, observamos que la consciencia de identidad (dimensión interna) no emerge hasta el momento en que aparece un "otro" (dimensión externa) del cual diferenciarse. Nacemos con etiquetas ligadas a grupos de pertenencia y categorías sociales con las que nos identifican y hemos de identificarnos, $y$ llegamos a percibirnos e identificarnos a nosotros mismos como los demás lo hacen.

Es por ello que para Mead (1974) el self o sí mismo es el resultado del proceso de interacción de dos componentes. Por un lado está el yo como agente activo y por otro el "mi" (me), o sí mismo, como objeto, que resulta de la interiorización de las actividades y funciones del yo organizadas por los otros, es decir, los roles que el sujeto debe ejercitar a lo largo de su vida. Para Yuval-Davis (2010), las identidades deberían ser entendidas como formas específicas de narrativas relativas al yo y a sus límites. En su investigación muestra cómo las narrativas sobre la identidad son inevitablemente sobre los límites y las definiciones del "yo" y del "no yo". Los otros no son siempre personas concretas que afectan al individuo en interacciones cara a cara. Existe un "otro generalizado" como marco en el que la persona se inscribe, como grupo o comunidad que proporciona al individuo un referente frente al que organizar su peculiaridad. Desde esta perspectiva, el alter es un otro generalizado, otro encarnado en toda una comunidad en la que el individuo se encuentra inserto. Este otro generalizado funciona como un marco de valores y símbolos compartidos con los que la persona se evalúa y contrasta, y que a través del "mi” llega a formar parte de la propia identidad. No obstante, a pesar de ser una idea muy extendida, no existen estudios que hayan medido de forma empírica la presencia de esos "otros" en discursos sobre la propia identidad, ni del alter en la narrativa del yo, lo cual intentamos enfrentar en este trabajo.

En un estudio sobre la inmigración peruana en Madrid, Labrador (2001) señala cómo la identidad personal de los inmigrantes es afectada por la mirada del "otro", en este caso la comunidad española que recibe a los inmigrantes y que transfiere a cada individuo categorizado como tal la posición de "inmigrante trabajador con poca cualificación y que debe someterse a todo los que se le imponga". El inmigrante termina en parte reflejando en su propia actuación (su "yo" en palabras de Mead) el estigma (el "mí") derramado en él o ella por parte de la sociedad receptora (otro generalizado). De un modo general, podemos decir que toda igualdad nos introduce necesariamente en el concepto de diferencia. Siempre que se establece una relación de similitud entre dos objetos es porque existe también una de diferencia, es decir que hay unos objetos que no son incluidos en la relación de similitud.

Esta misma tensión identidad-diferencia se aprecia en la construcción de la identidad de grupos. Hall (1990) habla de dos modos de pensar sobre la identidad cultural. El primero define la identidad cultural en términos de una cultura compartida, pero es evidente que existe una enorme heterogeneidad interna en el grupo cultural definido como idéntico y límites imprecisos entre grupos. Esta heterogeneidad es señalada por estudios que enfocan la identidad cultural desde una visión diferente, aunque complementaria, a la hasta aquí expuesta. Esta segunda posición reconoce que además de muchos puntos de semejanza, existen puntos de diferencia críticos que constituyen aquello que realmente somos. No podemos hablar de una identidad, de una continuidad y una unicidad, sin tener en cuenta la otra cara de la moneda, las 
rupturas y las diferencias que constituyen tal unicidad. Desde este segundo sentido, la identidad cultural es más un hecho de hacerse o convertirse en, que de ser, que pertenece al futuro además de al pasado.

Desde la perspectiva de la psicología social, Jenkins (1997) ofrece una versión simplificada de las teorías de la diferenciación social (Tajfel, 1984) y la de la categorización social (Turner, 1991), integrando ambas en una explicación común. Este autor diferencia entre el nivel colectivo de la identificación grupal (colectividad que se identifica y define a sí misma) y el de la categorización social (una colectividad que es identificada y definida por otros). La identificación grupal es presentada como el momento interno de la identificación, mientras que la categorización social es el momento externo. La identidad colectiva surge entonces de la dialéctica entre estos dos momentos, el interno y el externo. La identidad de un grupo o identidad social es construida a través de sus límites en la interacción con otros en un contexto social (Vallejos, Wigstrom, Olivares, Yzerbyt \& Estrada, 2015).

El estudio de la identidad nacional ha gozado de cierta preferencia entre las distintas identidades sociales. Muchas de las investigaciones realizadas sobre las representaciones sociales tienen el referente de la Nación. Por ejemplo, se analiza cómo las personas adquirimos una identidad como miembros de nuestra Nación, una identidad nacional (Smith, 1991). Esta ha sido definida como el conjunto de "identificaciones y representaciones relativamente estables en el tiempo que permiten a los miembros de un grupo social que comparten una historia y un territorio común, así como otros elementos socio-culturales, reconocerse como relacionados los unos con los otros, biográficamente" (Montero, 1984, p. 26).

La pertenencia a nuestro grupo nacional nos lleva a definir nuestra identidad nacional, a etiquetarnos a nosotros mismos como españoles, rusos o franceses. Unida a esta existe una representación social de lo que significa ser español, ruso o francés. La identidad nacional está relacionada con la manera en la que una persona ve a su país, con lo que para ella significa ser, por ejemplo, español, al cual abarca el conjunto de creencias, estereotipos y actitudes caracterizadas por un sentido de consciencia nacional. Dicho de otra manera, consiste en un sentido de pertenencia a un grupo, ficticio o natural, que tiene una memoria cultural e histórica $\mathrm{y}$ un conjunto de costumbres cotidianas.

Desde otra perspectiva la identidad estaría mejor definida por sus fronteras (Barth, 1976). Según esta corriente, mayoritaria actualmente en los estudios antropológicos sobre la identidad, esta se construye en contraposición con "el otro", y ese otro puede encontrarse en distintos niveles de diferenciación, dependiendo de en qué momento y para qué actividad nos definamos como iguales o desiguales. Por ejemplo, en un estudio de campo Shweder (1990) mostró cómo personas que aceptan clasificarse dentro de un mismo grupo para elegir a un representante nacional pueden no hacerlo para otras actividades como compartir familia o escuela.

Las fronteras, por tanto, no son algo tajante y predeterminado, sino más bien un límite cambiante usado por los individuos o los grupos para definir sus diferencias o semejanzas con otros grupos dependiendo del escenario de actividad en el que se enmarcan sus interacciones. En palabras de Pujadas (1993), "La identidad es inseparable de la otredad: Los individuos y los pueblos conciben su identidad frente a la alteridad" (p. 9).

De esta forma, desde nuestra perspectiva la identidad es un concepto funcional que las personas "usan" en una situación y marco de actividad determinado. La identidad cultural, por su parte, surge en la consciencia y se hace reivindicación en un grupo en el momento en que se hace útil para la consecución de uno o varios objetivos políticos, sociales o económicos. Con esta definición, más cercana a las posturas situacionistas interesadas en el análisis de los límites culturales, intentamos escapar del carácter estático y homogeneizador 
que en ocasiones acarrea el mismo concepto de identidad cultural.

No consideramos, pues, la identidad una esencia inmutable y transituacional, sino un concepto cuya construcción por parte de las personas viene conformado por los contextos de interacción en los que estas se desenvuelven y en los que el alter juega un papel clave. También el caso de la identidad andaluza (comunidad autónoma del Sur de España) puede ilustrar los procesos de emergencia de la consciencia de la identidad en situaciones de alteridad.

Como explica Moreno (1992), la emigración andaluza produjo una considerable toma de consciencia de su propia idiosincrasia por parte de la comunidad andaluza. A partir de los años sesenta se produce una masiva emigración de andaluces hacia el resto del Estado español, especialmente a Cataluña, y hacia Europa. En este momento es cuando se empieza a cristalizar la autoconsciencia de etnicidad. Andalucía en esta época pasa a ser, además de una región que tradicionalmente exporta materias primas, una exportadora de mano de obra. Y en Cataluña, el País Vasco o Alemania, cuando se enfrentan a una realidad distinta, los andaluces empiezan a ser conscientes de las contradicciones que se dan en su tierra, con grandes potencialidades pero con una economía pobre. Empiezan a verse como portadores de una cultura propia y diferenciada. Al ser la emigración andaluza un fenómeno notable en la época citada, este acontecimiento produce efectos en la comunidad andaluza como tal, tanto por la propia experiencia, como por la experiencia vicaria de familiares, amigos o conocidos. Esta es una experiencia de confrontación que se extiende a toda la comunidad andaluza y da lugar a una importante emergencia de la identidad cultural colectiva que afecta a la comunidad más allá de los sujetos individuales que emigraron (Macías-Gómez-Estern, 2013).

En esta introducción hemos intentado ubicar de forma general las distintas teorías sobre los procesos identitarios que se han desarrollado en las ciencias sociales. La perspectiva adoptada por estos estudios se sitúa mayoritariamente en un plano macrosocial de análisis. Sin embargo, cabe preguntarse ¿en qué medida y de qué manera afectan los procesos políticos y sociales a los sujetos que habitan y son socializados en un marco nacional concreto?, ¿cómo construyen estas personas su identidad cultural? Y, especialmente, ¿qué papel juega el otro en el discurso sobre la propia identidad?

A pesar de los múltiples indicios de la importancia de la figura del alter como objeto de comparación y espejo ante el que se construye el yo, no existen investigaciones que analicen este hecho de forma empírica. Consideramos que se hace necesario el establecimiento de diseños de investigación y procedimientos analíticos que puedan aportar evidencias a este respecto, y tenemos la hipótesis de que aquellas personas que han pasado por procesos de migración, de una u otra forma, son las que mejor podrán reflejar en sus discursos identitarios la presencia del otro.

Por estos motivos, el objetivo que nos planteamos en esta investigación fue analizar las narrativas respecto a la identidad andaluza de personas con diferentes experiencias de migración, no para aportar un nuevo intento de definición de esta, sino para comprobar la presencia del alter en los discursos. Partimos de la hipótesis de que aquellas personas con experiencia directa de migración presentarán un mayor número de referencias directas a otras personas o referentes de comparación. De quienes no cuentan con dicha experiencia esperamos que presenten comparaciones o referencias de tipo genérico, es decir, menciones a personas no definidas. Mientras, esperamos encontrar en el grupo de emigrantes retornados una posición intermedia entre los otros dos grupos, puesto que si bien tienen experiencia migratoria y, por tanto, cuentan con una mayor cantidad de recursos e información a compartir, han vuelto y en la actualidad viven en Andalucía. 


\section{Método}

El diseño de este trabajo es descriptivo y se maneja la "experiencia de emigración", entendida como experiencia de confronte con un alter cultural como la variable explicativa. La construcción de la identidad cultural fue considerada la variable criterio, que se operativizó en un sistema de categorías de elaboración propia en el que se analizaban la estructura y el contenido de las narrativas de identificación. En este trabajo ponemos el foco en una de las categorías que analizan la estructura del discurso identitario: perspectiva del hablante, la cual está centrada en el uso como objeto o como referente de comparación del "otro cultural" en la construcción identitaria. La unidad que consideramos de análisis fue el enunciado, entendido como el turno de palabra de cada participante, la intervención sin interrupciones en el argumento de cada uno. Para más información sobre la metodología del estudio completo y las otras variables consideradas, veáse Macías-Gómez-Estern, García y Sánchez (2008) o Macías-Gómez-Estern, Amián, Sánchez y Marco (2010).

\section{Participantes}

La muestra estuvo compuesta de 34 participantes andaluces adultos (16 mujeres y 18 hombres), distribuidos equitativamente según tres condiciones: personas que no han emigrado de Andalucía ni tenían familiares directos migrantes (no emigran- tes), personas que emigraron y retornaron (emigrantes retornados) y personas que se encontraban viviendo en ese momento fuera de Andalucía, concretamente en Madrid (emigrantes).

La muestra fue buscada por distintas vías. Primero, en el caso de los emigrantes residentes en Madrid, se contactó con dos de las Casas de Andalucía que existen allí. En ambos casos el nivel socioeconómico de la mayoría de los participantes era medio y medio-bajo, que equivalía al perfil típico de trabajador poco cualificado (Moreno, 1993). El grupo de los emigrantes retornados fue contactado a través de la Asociación Sevillana de Andaluces Retornados, que facilitó una lista de nombres y teléfonos de personas adscritas a esta. La parte de la muestra que nunca emigró fue recopilada por contactos personales. Debido a que la primera muestra era la más difícil de encontrar, se buscó de forma intencional a participantes con las mismas características que las del primer grupo para que, tal como se muestra en la tabla 1, se pudieran considerar grupos equiparados en género, nivel educativo y edad.

\section{Procedimiento}

La recogida de datos se llevó a cabo mediante la realización de debates, con la técnica de grupos de discusión, entre los participantes pertenecientes a cada una de las categorías señaladas (no emigrantes, emigrantes retornados y emigrantes). Antes de realizar las sesiones de discusión definitivas,

Tabla 1

Distribución de la muestra por género, edad y nivel educativo

\begin{tabular}{lccccccc}
\hline & \multicolumn{2}{c}{ Género } & Edad & \multicolumn{2}{c}{ Nivel educativo } \\
\cline { 2 - 7 } & F & M & & No estudios & Primario & Secundario & Superior \\
\hline No emigrantes & 6 & 6 & $25-61$ & 1 & 5 & 2 & 4 \\
Emigrantes retornados $^{1}$ & 5 & 6 & $25-68$ & 1 & 3 & 3 & 4 \\
Emigrantes $^{1}$ & 5 & 6 & $24-73$ & 3 & 5 & 1 & 3 \\
\hline
\end{tabular}

${ }^{1}$ Tanto los emigrantes como los retornados habían estado fuera de Andalucía más de 10 años en todos los casos, y eran equivalentes en cuanto al destino, y habían vivido en Madrid, Cataluña o en otros países centroeuropeos. 
se llevaron a cabo cuatro debates piloto. La realización del pilotaje de estos permitió establecer el procedimiento a seguir y el manejo de los instrumentos a emplear durante las sesiones de discusión. Además, se destacaron algunos temas recurrentes que se usarían posteriormente tanto para iniciar el debate como para mantener una dinámica fluida. Los temas que se identificaron como más recurrentes fueron los siguientes: economía, política, gastronomía, folclore, clima, costumbres y forma de ser o personalidad.

Se llevaron a cabo dos grupos de discusión por cada condición, siendo el número de participantes de entre cinco y seis personas en cada uno. Se realizaron, por tanto, seis debates en total, que se llevaron a cabo en un seminario de la Facultad de Psicología de Sevilla (Andalucía, España) o en los locales de la Casa de Andalucía de otras ciudades. La situación perseguida pretendía simular las discusiones cotidianas en las que puedan aparecer discursos en torno a la identidad, por tanto, se creaba o se incitaba una discusión de tales características. Antes de comenzar se solicitó y obtuvo firmado el consentimiento informado de cada uno de los sujetos a participar en la investigación.

Cada uno de estos debates fue registrado en vídeo, colocando la cámara de modo que interfiriera lo menos posible en la situación. Antes de iniciar la discusión se advertía a los participantes del uso exclusivo de la grabación por parte de los investigadores para el trabajo que aquí se expone. También se enfatizó que lo que se pretendía era conocer opiniones, no respuestas verdaderas, sobre el tema andaluz. Se les comentó brevemente en qué consistía el estudio, y se enfatizó que el objetivo era acceder a los puntos de vista de distintas personas, no a sus conocimientos, y mucho menos pretendíamos evaluarlos o juzgarlos.

Se pidió a los participantes que discutieran entre ellos sobre la pregunta de si existe o no una identidad andaluza. Por lo tanto, debían argumentar su opinión sobre si hay rasgos diferenciales que hagan de lo andaluz algo genuino o no. Para animar la discusión e instigar el debate, la investigadora presentó al grupo, simplemente citándolas y sin desarrollar argumento alguno, las dos posturas opuestas mayoritarias al respecto (existe una identidad andaluza y no existe una identidad andaluza).

Cada uno de los seis debates realizados tuvo una duración de entre veinte minutos y una hora. Las grabaciones de estos fueron después transcritas y codificadas según un sistema de categorías de elaboración propia, analizándose solo los 100 primeros enunciados de cada uno de ellos, puesto que era el número máximo de enunciados en torno a la pregunta formulada que compartían todos los debates.

\section{Codificación}

Para codificar la perspectiva del hablante en su discurso, se analizó el punto de vista del sujeto, la situación de partida sobre la que construye el enunciado y se codificó cada "narrativa de identificación". Incluimos en la tabla 2 los diferentes códigos empleados, su definición y un ejemplo literal extraído de las transcripciones de los debates.

La primera de las categorías consideradas fue comparación. Se consideró que hubo una comparación cuando el participante para describir, explicar o narrar utiliza como referente a otra persona o colectivo de personas con el que se contrasta. En el contexto del debate, el hablar de otro colectivo sin nombrar a lo andaluz implica que se está utilizando la comparación ya que este se presupone. Las comparaciones codificadas previamente fueron además codificadas de nuevo en función de su objeto y referente de comparación, y el grado de explicitud en el que estos aparecen. Decidimos excluir de este análisis tanto las 83 intervenciones (13.83\% del total) relativas a la propia dinámica del debate, como las preguntas a los investigadores sobre las normas, la tarea o cualquier otra cuestión ajena al tema de interés. 
El objeto de la comparación referido se consideró aquel elemento que es tomado como el núcleo de esta, aquello que se está describiendo que puede ser local, regional, nacional o supranacional. Por su parte, el referente de la comparación es el elemento que es tomado como contraste para calificar al objeto que también puede ser local, regional, nacional, supranacional o indefinido. Estos últimos eran referencian a un otro generalizado, por ejemplo, "Los andaluces somos diferentes a las gentes de fuera". El grado de explicitud del objeto de la comparación se refiere a cuán explícito el participante hace el objeto de su comparación, y el grado de explicitud del referente de la comparación se refiere a cuán explícito el participante hace el referente de su comparación.

Tabla 2

Sistema de codificación para los debates sobre la identidad andaluza

\begin{tabular}{|c|c|c|c|}
\hline Variables & Valores & Definición & Ejemplos \\
\hline \multirow{2}{*}{ Comparación } & No realiza comparación & No toma a otros como referente & $\begin{array}{l}\text { "[...] nosotros somos tan trabajadores co- } \\
\text { mo ellos, y, además, sabemos divertirnos". }\end{array}$ \\
\hline & Realiza comparación & $\begin{array}{l}\text { Toma a otros como referente } \\
\text { para construir el enunciado }\end{array}$ & "El andaluz es tolerante". \\
\hline \multirow{3}{*}{$\begin{array}{l}\text { Objeto de la } \\
\text { comparación }\end{array}$} & Local & $\begin{array}{l}\text { El elemento descrito es una } \\
\text { unidad local }\end{array}$ & $\begin{array}{l}\text { "En Sevilla somos más simpáticos que } \\
\text { allí". }\end{array}$ \\
\hline & Regional & $\begin{array}{l}\text { El elemento descrito es una uni- } \\
\text { dad regional }\end{array}$ & “Andalucía es el lugar más bonito". \\
\hline & $\begin{array}{l}\text { Nacional o suprana- } \\
\text { cional }\end{array}$ & $\begin{array}{l}\text { El elemento descrito es una uni- } \\
\text { dad nacional o supranacional }\end{array}$ & $\begin{array}{l}\text { "En España la economía no va tan bien } \\
\text { como en Europa". }\end{array}$ \\
\hline \multirow{4}{*}{$\begin{array}{l}\text { Referente de la } \\
\text { comparación }\end{array}$} & Local & $\begin{array}{l}\text { El elemento de referencia es una } \\
\text { unidad local }\end{array}$ & $\begin{array}{l}\text { "En Sevilla somos más simpáticos que los } \\
\text { de Madrid". }\end{array}$ \\
\hline & Regional & $\begin{array}{l}\text { El elemento de referencia es una } \\
\text { unidad regional }\end{array}$ & $\begin{array}{l}\text { "Andalucía es lugar más bonito geográfica- } \\
\text { mente que Cataluña". }\end{array}$ \\
\hline & $\begin{array}{l}\text { Nacional o suprana- } \\
\text { cional }\end{array}$ & $\begin{array}{l}\text { El elemento de referencia es una } \\
\text { unidad nacional o supranacional }\end{array}$ & $\begin{array}{l}\text { En España la economía no va tan bien co- } \\
\text { mo en Europa". }\end{array}$ \\
\hline & Indefinido & $\begin{array}{l}\text { El elemento de referencia de la } \\
\text { comparación es un otro gene- } \\
\text { ralizado }\end{array}$ & $\begin{array}{l}\text { "Los andaluces somos diferentes a las gen- } \\
\text { tes de fuera". }\end{array}$ \\
\hline \multirow{2}{*}{$\begin{array}{l}\text { Grado de expli- } \\
\text { citud del objeto } \\
\text { de la compara- } \\
\text { ción }\end{array}$} & Explícito & $\begin{array}{l}\text { El participante señala explíci- } \\
\text { tamente cuál es el objeto de su } \\
\text { comparación }\end{array}$ & $\begin{array}{l}\text { "En Sevilla somos más simpáticos que los } \\
\text { de Madrid". }\end{array}$ \\
\hline & Implícito & $\begin{array}{l}\text { El participante se refiere al ob- } \\
\text { jeto de su comparación con un } \\
\text { pronombre, adverbio de lugar u } \\
\text { omisión }\end{array}$ & "Se vive mejor que en Alemania". \\
\hline \multirow{2}{*}{$\begin{array}{l}\text { Grado de ex- } \\
\text { plicitud del } \\
\text { referente de la } \\
\text { comparación }\end{array}$} & Explícito & $\begin{array}{l}\text { El participante señala explícita- } \\
\text { mente cuál es el referente de su } \\
\text { comparación }\end{array}$ & $\begin{array}{l}\text { "En Sevilla somos más simpáticos que los } \\
\text { de Madrid". }\end{array}$ \\
\hline & Implícito & $\begin{array}{l}\text { El participante se refiere al refe- } \\
\text { rente de su comparación con un } \\
\text { pronombre o adverbio de lugar } \\
\text { u omisión }\end{array}$ & $\begin{array}{l}\text { "Aquí se vive mejor que allí". } \\
\text { "En Andalucía se vive mejor". }\end{array}$ \\
\hline
\end{tabular}




\section{Resultados}

En la tabla 3 se presenta un análisis exploratorio de las codificaciones realizadas en las cinco categorías consideradas.

Tabla 3

Resultados exploratorios de la perspectiva del hablante

\begin{tabular}{lcc}
\hline \multicolumn{1}{c}{ Comparación } & Frecuencia & Porcentaje \\
\hline No compara & 206 & 39.85 \\
Compara & 311 & 60.15 \\
& 517 & 100.0 \\
\hline
\end{tabular}

\begin{tabular}{lcc}
\hline \multicolumn{3}{c}{ Objeto de la comparación } \\
\hline Local & 38 & 12.3 \\
Regional & 242 & 77.7 \\
Nacional/supranacional & 31 & 10.0 \\
& 311 & 100.0 \\
\hline
\end{tabular}

\begin{tabular}{lcc}
\hline \multicolumn{3}{c}{ Referente de la comparación } \\
\hline Local & 107 & 34.4 \\
Regional & 95 & 30.5 \\
Nacional/supranacional & 73 & 23.5 \\
Indefinido & 36 & 11.6 \\
& 311 & 100.0 \\
\hline
\end{tabular}

Grado de explicitud del objeto de la comparación

\begin{tabular}{lcc}
\hline Explícito & 149 & 47.9 \\
Implícito & 162 & 52.1 \\
& 311 & 100.0 \\
\hline
\end{tabular}

Grado de explicitud del referente de la comparación

\begin{tabular}{lcc}
\hline Explícito & 139 & 44.7 \\
Implícito & 172 & 55.3 \\
& 311 & 100.0 \\
\hline
\end{tabular}

Los análisis llevados a cabo a través de la prueba chi-cuadrado muestran cómo existe una variación en el uso de comparaciones en el discurso sobre identidad en relación con la experiencia de emigración de los participantes $\left(\chi^{2}:=15.673 ; p<0.01\right)$. Los emigrantes retornados muestran valores no significativos con un residual estándar ajustado
—REA— de 0.8. No obstante, los residuales ajustados estandarizados de los niveles "No compara" y "Compara" son significativos en los no emigrantes y los emigrantes ( 2.9 y 3.8 , respectivamente). Los primeros comparan menos de lo esperado, según un modelo de distribución equiprobable, y los segundos muestran la tendencia inversa.

De modo general, y tal como se muestra en la figura 1, podemos decir que la experiencia de emigración hace que los sujetos de comparen más en sus narrativas de identificación en los debates que las personas que no han emigrado.

Una vez analizadas las diferencias en la cantidad de comparaciones con un "otro" usadas por los tres grupos de participantes, examinaremos en el siguiente apartado quién o quiénes son esos "otros" y qué tan presentes se encuentran en el discurso de los participantes en nuestro estudio. Esta es la función que cumplen las categorías "objeto de la comparación", "referente de la comparación" y el grado de explicitud que presentan.

\section{Análisis del objeto de comparación}

En primer lugar, podemos observar cómo la relación entre la experiencia de emigración y objeto de la comparación obtiene un $\chi^{2}=62.07$ y una $p<0.01$. Dicha prueba mostró que los residuales ajustados estandarizados de todas las casillas, salvo la de lo regional para los no emigrantes y la de lo local para los emigrantes, obtienen valores significativos. Los datos muestran una clara tendencia en los no emigrantes a usar lo local como objeto de sus comparaciones más de lo esperado, según un modelo de equiprobabilidad $(\mathrm{REA}=3.4)$. También observamos una tendencia significativa en este grupo a usar menos de lo esperado un objeto de comparación nacional o supranacional $($ REA $=-2.5)$. Algo similar ocurre en el grupo de emigrantes, el cual también usa menos de lo esperado lo nacional o supranacional como objeto de sus comparaciones ( $\mathrm{REA}=-4.3)$. En este nivel, no emigrantes y emigrantes se diferencian 


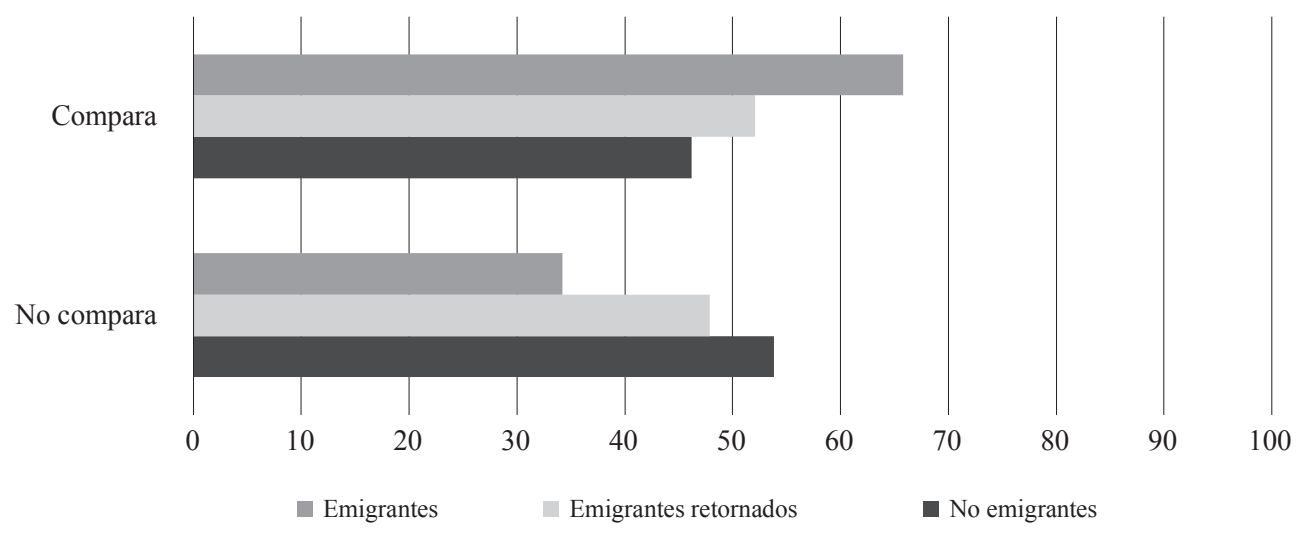

Figura 1. Experiencia de emigración por comparación. $\chi^{2}$ : $15.673 ; p<0.01$

claramente de los emigrantes retornados, quienes rotundamente usan más de lo esperado el objeto de comparación nacional o supranacional (REA =7.0), y que significativamente usan menos de lo esperado tanto el objeto de comparación local $($ REA $=-3.7)$ como el regional $($ REA $=-2.1)$. Los emigrantes actuales usan mayoritariamente lo regional como objeto de su comparación $(\mathrm{REA}=2.8)$, siendo esta diferencia muy significativa.
De modo general, y tal como se muestra en la figura 2, podemos decir que los grupos de no emigrantes y de emigrantes tienden a usar un objeto local para sus comparaciones, en cierta medida, y especialmente el regional, lo que retomaremos en la discusión. Sin embargo, en comparación con los emigrantes retornados, aparece escasamente en sus comparaciones el objeto nacional. Contrariamente, los emigrantes retornados usan más de lo esperado el

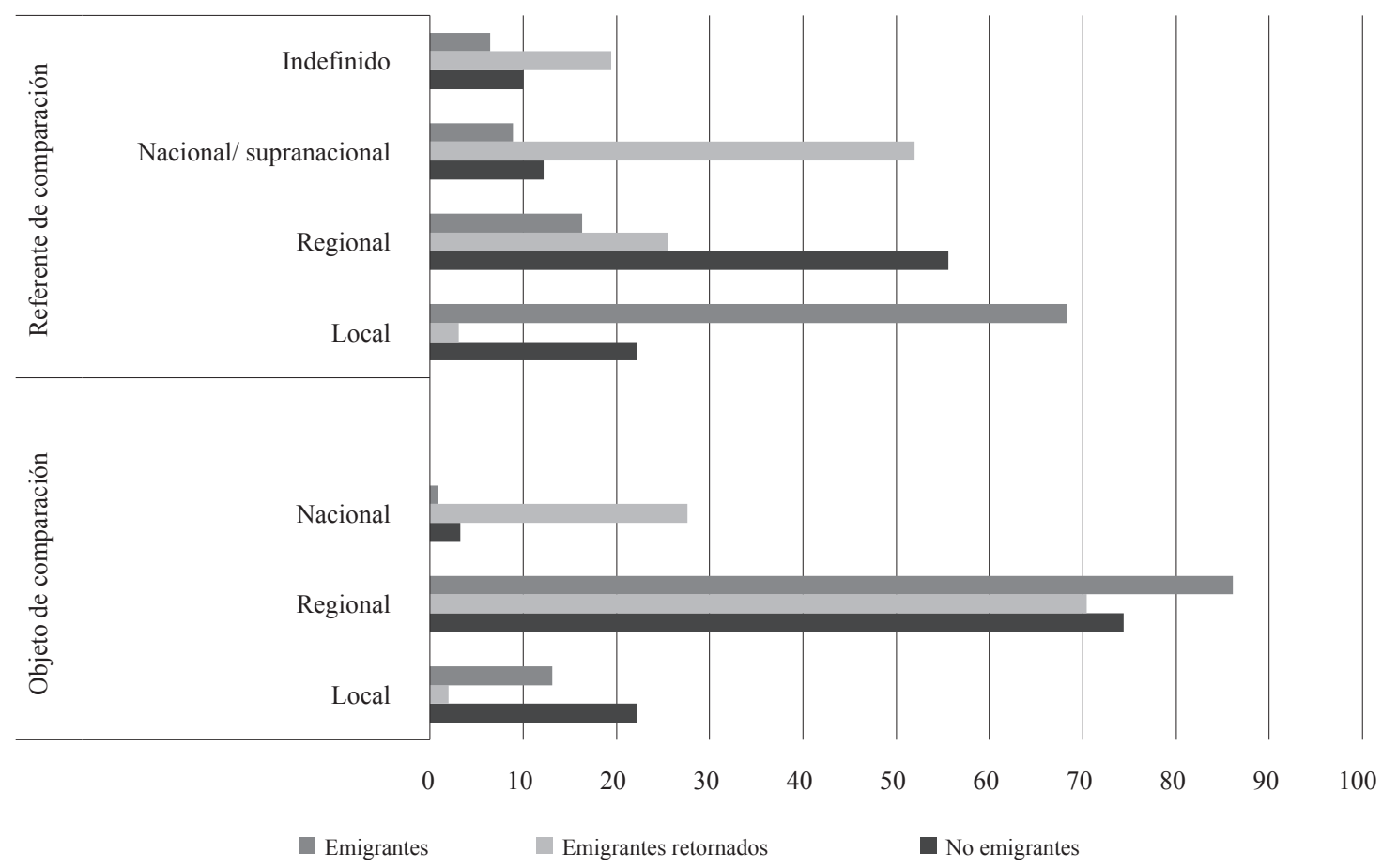

Figura 2. Relación entre la experiencia de emigración y el objeto y referente de comparación 
objeto de la comparación nacional o supranacional, siendo lo local y lo regional objetos de comparación muy poco empleados por este grupo.

\section{Análisis del grado de explicitud del objeto de comparación}

Por otra parte, podemos observar cómo la relación entre la experiencia de emigración y el grado de explicitud del objeto de la comparación obtiene un $\chi^{2}=13.56$ con una $p<0.01$. En la figura 3, se observa que los no emigrantes y los emigrantes son igualmente explícitos. En ambos casos esta relación presenta un residual ajustado estandarizado de 1.8, no estadísticamente significativo, mientras que los emigrantes retornados sí son explícitos al señalar el objeto de sus comparaciones, siendo este valor mayor de lo esperado según un modelo equiprobable ( REA $=3.7)$. En la figura 3, se observa una tendencia asimétrica en la presentación implícita o explícita del objeto de las comparaciones en cuanto a los perfiles de los participantes. Los no emigrantes y emigrantes tienden a hacer presentaciones implícitas, mientras que los emigrantes retornados tienden en mayor medida a hacer una presentación explícita del objeto de comparación.

\section{Análisis del referente de comparación}

Podemos observar cómo la relación entre la experiencia de emigración y el referente de la comparación obtiene un $\chi^{2}=158.46$ con un $p<0.01$. Dicho análisis reflejó que 10 de los residuales ajustados estandarizados presentaron valores significativos. En este caso, tanto no emigrantes como emigrantes retornados usan lo local menos de lo esperado como referente de sus comparaciones, según un modelo de equiprobabilidad. Los emigrantes, sin embargo, sí usan lo local como referente de sus comparaciones más de lo esperado, mientras que usan menos de lo esperado todos los demás referentes de comparaciones. Con respecto a lo regional como referente de la comparación, son los no emigrantes los que lo usan más de lo esperado. Por su parte, los emigrantes retornados usan lo nacional y lo supranacional como el referente más de lo esperado. Los únicos indicadores en los que no se obtuvieron diferencias significativas fueron en el caso de indefinidos para los no emigrantes $(\mathrm{REA}=-0.6)$ y el referente local para los emigrantes retornados $(\mathrm{REA}=-1.3)$.

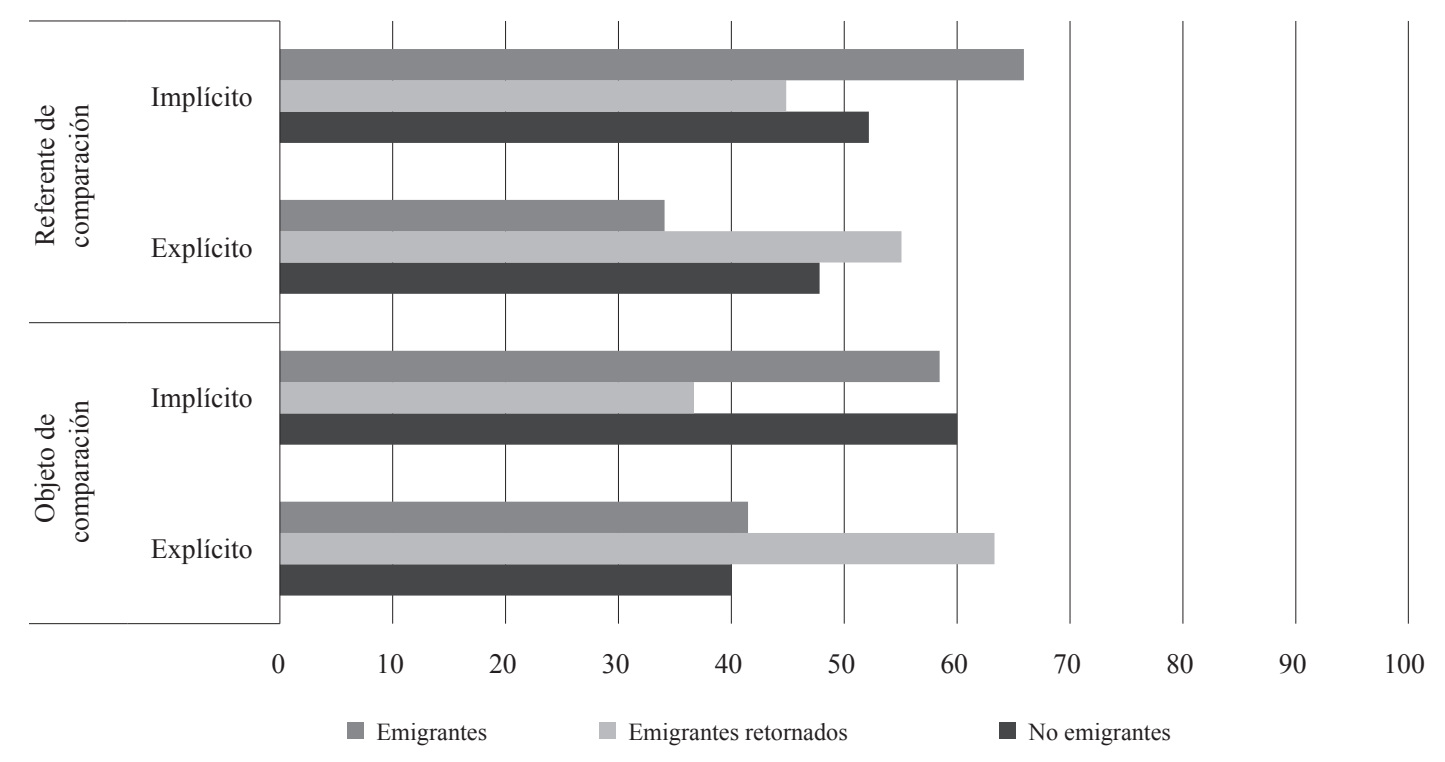

Figura 3. Relación entre la experiencia de emigración y el grado de explicitud del referente y el objeto de comparación 
En la figura 2 se ilustran las relaciones descritas. Se observa que los referentes de comparación más generales (regional, nacional o supranacional e indefinido) son poco usados por los emigrantes a diferencia de los emigrantes retornados, que se valen del referente nacional o supranacional y el indefinido más de lo esperado. Contrariamente, el empleo de lo local como referente de la comparación obtiene valores inversos a lo supranacional e indefinido en los emigrantes y emigrantes retornados, siendo los primeros los que lo usan más de lo esperado mientras que los segundos apenas lo emplean. Los no emigrantes usan otras regiones como referente más de lo esperado.

\section{Análisis del grado de explicitud del referente de comparación}

Por último, la relación entre la experiencia de emigración y el grado de explicitud del referente de la comparación obtiene un $\chi^{2}=10.18$ y una $p<0.01$. Los residuales ajustados estandarizados de los no emigrantes (0.7) no muestran diferencias significativas. Sin embargo, en los emigrantes $($ REA $=3.0)$ sí se observó que hacía muchas más referencias implícitas que explícitas, y en los emigrantes retornados $($ REA $=2.5)$ se observó la tendencia contraria, la de explicitar el referente de sus comparaciones más de lo esperado según un modelo de equiprobabilidad.

En la figura 3 se refleja la relación asimétrica que se da entre los emigrantes y los emigrantes retornados en el grado de explicitud con que presentan el referente de sus comparaciones. Mientras que los primeros son muy implícitos cuando se refieren al alter de sus comparaciones, los segundos son mucho más explícitos en el referente de sus comparaciones.

Tal como hemos podido observar, todos los análisis llevados a cabo mediante la prueba chi-cuadrado muestran que existen múltiples variaciones significativas en el tipo de comparaciones usadas en el discurso sobre identidad en relación con la experiencia de emigración de los/las participantes. De esta forma se ha observado que esta relación es significativa en los cuatro códigos considerados.

\section{Análisis de tres extractos de los debates}

A continuación, y para finalizar este apartado, se presentan tres extractos de los debates realizados. Consideramos que son buenos ejemplos, entre otras cuestiones, de las intervenciones y de cómo en estos escenarios surgieron cuestiones muy personales sobre la propia identidad. De hecho, se puede observar fácilmente cómo en aquellos en los que había personas que habían emigrado durante mucho tiempo surgían ejemplos de la vida personal y narrativas biográficas para ejemplificar la identidad andaluza en su conjunto:

Debate 6. Emigrantes. Enunciado 4. Hablante: Loli.

Entonces yo era una persona muy feliz, éramos cuatro de familia, mis padres mi hermano y yo. Mi padre era un trabajador nato, por circunstancias de la vida mi hermano se vino a Madrid, y bueno, con una locura impresionante de que nos viniéramos todos. Yo no quería venirme, pero bueno, esa ilusión de siempre de la capital de España. Yo me siento inmigrante, pero con un dolor muy grande porque yo soy andaluza por los cuatro costados. Entonces, si yo volviera ahora otra vez con mis raíces, bueno, al pueblo me iba con los ojos cerrados.

En los debates de emigrantes, como el número 6, el objeto de las comparaciones es Andalucía o los andaluces como comunidad, que se narra de un modo implícito, de manera que se considera conocido por el auditorio. Sin embargo, en los de no emigrantes, como el siguiente, se usaban más de forma implícita los objetos de comparaciones locales: 
Debate 1. No emigrantes. Enunciado 5. Hablante: Ramón.

Es la mentalidad, el andaluz tiene una especie de personalidad diferente. [...] Personas que podemos, por ejemplo, nos tomamos la vida un poco menos dramática, aunque luego tengamos dentro nuestra cosa. Y dicen que menos seria, pero no, el andaluz es serio.

Finalmente, los emigrantes retornados, a diferencia de los anteriores, han usado más de lo esperado el objeto de la comparación nacional o supranacional (España o Europa) de un modo claramente explícito.

En relación con el referente de la comparación, encontramos que, de manera coherente con los objetos, en el caso de los emigrantes es local y altamente implícito; los no emigrantes usan un referente regional sin arrojar significatividad en el grado de explicitud expresado; los emigrantes retornados, por su parte, usan un referente nacional o supranacional de manera explícita:

Debate 3. Emigrantes retornados. Enunciado 23. Hablante: José.

Pero yo pienso una cosa. Yo creo que un error para nosotros mismos los andaluces es precisamente ese comentario que tú acabas de hacer: que vivimos muy bien. Efectivamente, aquí vive muy bien el que tiene trabajo o el que tiene una buena posición. Pero que hay gente que vive muy mal. Y hay que tener en cuenta en el contexto en el cual está Andalucía en relación a España. Que estamos [...], la comunidad autónoma creo que es la última. Sevilla concretamente, en términos generales está en el puesto número cuarenta de España. Seguimos bien, porque tocamos las palmas y porque van a hacer una romería o a la feria, y como tenemos nuestro carácter extrovertido [...] pues vamos a una romería o a la Semana Santa [...].

\section{Discusión}

Con objeto de conocer la relación entre los diferentes grados de experiencia de migración y la forma en la que se entiende y explica la identidad andaluza, se han estudiado, a través del análisis narrativo de grupos de debate, los discursos respecto a la identidad andaluza que tienen personas que han migrado y retornado, que han migrado y no han retornado, y que nunca han migrado.

Si hay resultado destacable de los diferentes análisis realizados es la crucial importancia que la experiencia de migración tiene en la consideración del "otro", que aparece en un $62.4 \%$ de los enunciados considerados en los que se debate sobre la identidad andaluza. Sin duda, aparecieron un número significativamente mayor de referencias a ese alter cuando los debates se realizaron entre personas con experiencia de migración que en los debates de personas sin dicha experiencia. Este dato muestra la relevancia de la migración como vivencia de alteridad en la construcción de la identidad cultural con respecto a la comunidad de origen, como desarrollamos en la introducción (Clegg y Salgado, 2011; Mead, 1974; Labrador, 2001; Yuval-Davis, 2010).

Es importante destacar, en primer lugar, que el $77.7 \%$ del total de los enunciados en los que hay comparación se concentra en el valor regional (tabla 3), lo cual puede estar influido en cierta medida por el modo en que se ha planteado el debate (la existencia o no de la identidad andaluza). En esta discusión, además de profundizar en el análisis de los resultados cuantitativos, reflexionaremos sobre los extractos presentados. Un análisis cualitativo más pormenorizado de los datos presentados en este trabajo, en combinación con otras categorías del discurso, se puede encontrar en Macías-Gómez-Estern et al. (2008), Macías-Gómez-Estern et al. (2010), Macías-Gómez-Estern y De la Mata (2013) y en Macías-Gómez-Estern (2013). 
Si enfocamos al detalle cómo son las comparaciones usadas por los participantes en nuestra investigación, ejemplificadas en los extractos presentados, encontramos que el objeto de la comparación más usado por los emigrantes es el regional, mostrándose implícitamente. En el caso de nuestros participantes, esto se refiere a que el objeto de sus comparaciones es Andalucía o los andaluces como comunidad, que se narra de un modo implícito, de manera que se considera conocido por el auditorio. En estos enunciados se observa que los emigrantes andaluces, ante la instrucción del debate de argumentar sobre la existencia o no de la identidad cultural andaluza, recurren a su experiencia personal de emigración, que es detallada y relatada en formato de narración. Los hablantes, para convencer a su audiencia sobre su postura acerca de la identidad andaluza, usan su propia historia personal de emigración, desde su propia subjetividad y experiencia cotidiana.

Nuestros datos sobre el objeto y la referencia de la comparación son interesantes desde la perspectiva de los estudios identitarios y la relación entre esta y la alteridad. Por un lado, los objetos de comparación no son estables en una comunidad independientemente de la situación en que las personas que los usan y de la experiencia que acumulan en sus trayectorias. El objeto y la referencia de las comparaciones usadas para construir la identidad variará en función de la experiencia de los participantes. Los límites del yo, por lo tanto, varían en relación con quiénes sean el "otro", tal y como desarrollamos en nuestra introducción.

En el caso de nuestro estudio, el yo que emerge en los debates de los participantes emigrantes es el "andaluz", lo cual, como argumentamos previamente, puede estar influido en cierta medida por el modo en que se ha planteado el debate (la existencia o no de la identidad andaluza). No obstante, consideramos fundamental el hecho de que las personas participantes habitan en una comunidad autónoma española diferente a Andalucía: Madrid (que en este caso es simultáneamente comunidad y provincia, por lo que el referente aparece como "local"). En el caso de los emigrantes andaluces, el alter es el madrileño (local-regional), y su yo se conforma como regional. Es interesante señalar cómo ambos componentes de la comparación, el objeto (Andalucía y los andaluces) y el referente (Madrid y los madrileños) aparecen implícitamente en las narraciones de los emigrantes. Según autores como Vygotski (1993), entre otros, la información contenida como implícita en el discurso es aquella que se da por "supuesta", por compartida por el grupo, por lo que no requiere su enunciación. En el caso de nuestros emigrantes, tanto el objeto como el referente de sus comparaciones están tan "naturalizados" que consideran que no es necesario mencionarlos en las narraciones identitarias que desarrollan en el momento del debate.

El caso de los no emigrantes muestra también esta naturalización y relación con la alteridad de las construcciones identitarias. Su objeto, aunque es fundamentalmente regional, es más local que el de otros participantes, en este caso las provincias de Sevilla y Málaga, donde se realizaron los grupos de discusión, y también implícito. El referente es mayoritariamente regional, usando a otras comunidades autónomas como alter.

Finalmente, los emigrantes retornados, que en el caso de nuestra investigación lo hacían desde distintos países centroeuropeos, usan tanto objetos (España) como referentes (otros países o zonas geográficas supranacionales) que evocan a su propia experiencia. En este caso, se identifican como andaluces y especialmente españoles en comparación con los otros perfiles de los participantes, ya que su alter es significativamente más nacional o supranacional. A diferencia de los emigrantes y los no emigrantes, los emigrantes retornados son altamente explícitos al enunciar tanto el objeto como el referente de sus comparaciones. Esto se explica por la circunstancia de que cada uno de ellos retornaba de un país diferente, por lo que esta no era información que se diera por supuesta en la situación comunicativa del grupo de discusión. 
Consideramos que la migración es sin duda una de esas situaciones en las que los individuos y los grupos se enfrentan a una discontinuidad (Macías-Gómez-Estern \& De la Mata, 2013). Con la experiencia migratoria se producen disrupciones biográficas en términos de redefiniciones de la identidad, a la vez que se transforman y cambian las voces culturales y los referentes con los que construimos nuestras narrativas autobiográficas (Esteban-Guitart \& Vila, 2015). Los resultados de este trabajo apuntan a una clara influencia de la experiencia de emigración en la construcción de la identidad cultural, tal como lo muestran las diferencias entre emigrantes y no emigrantes.

Es especialmente llamativo el modo y la frecuencia en que los emigrantes comparan en sus relatos las prácticas de los andaluces y los originarios del lugar donde residen. La comparación con el otro es para ellos un recurso casi ineludible. Los rasgos físicos y geográficos de Andalucía, relacionados con el apego a la tierra, son un tema preferido por los emigrantes en sus comparaciones. Dichos rasgos son tratados con una gran carga emocional, que idealiza la "tierra perdida" y la extraña. Así, cuando vemos que la identidad asume e incorpora las fronteras del yo (Yuval-Davis, 2010), lo hace con base en su propia experiencia con el alter. De este modo, cobra particular importancia la forma en la que un grupo construye un "otro", ya que la alteridad no es un fenómeno puramente "hecho", sino que se construye en la psique y afectividad de los grupos sociales (Guerrero \& Cuadra, 2013). Un grupo construye un "otro" porque hay un "nosotros", a la vez que la idea de "nosotros" necesariamente plantea la idea de un "ellos". Los no emigrantes de nuestro estudio tendieron a usar un discurso general y descriptivo sobre Andalucía y los andaluces en sus argumentos sobre identidad, mientras que los emigrantes prefirieron usar narraciones extraídas de su experiencia de emigración, en las que comparaban distintas facetas de la vida en Andalucía y en Madrid, su lugar de residencia.
Sin duda, la forma en la que entendemos al otro para referir nuestra identidad es clave, por ejemplo, en el mismo discurso político. Sakki y Pettersson (2016) muestran cómo las representaciones del otro como un grupo de personas desviado, amenazador o enemigos internos están muy presentes en los argumentarios de partidos políticos muy determinados. Desde esta perspectiva, y en función de los datos analizados, pensamos que el estudio del lenguaje sobre emociones puede aportar importantes hallazgos en un abordaje integrado de los procesos identitarios, en general, y del funcionamiento de estos en situaciones de emigración, en particular. Por ello, y para concluir, expresaremos la necesidad de seguir en esta línea de estudios sobre los procesos identitarios, no solo por el interés intrínseco que este tema pueda suscitar, sino también por la aplicación que a los conocimientos generados sobre este se pueda dar en la actualidad, dado el incremento de movimientos migratorios en todo el planeta. En concreto, consideramos que la realización de investigaciones similares desde perspectivas y análisis cualitativos permitiría conocer esta realidad en mayor profundidad.

Entre las limitaciones de este trabajo está el hecho de no haber podido desplegar un análisis cualitativo en profundidad y haber realizado un muestreo intencional. Otra limitación estuvo en el uso de debates quizás demasiado breves, que se debían a las dificultades de los participantes sin experiencia migratoria en debatir sobre un tema que en cierta medida les resultaba poco relevante.

Los resultados de este trabajo apuntan a una clara influencia de la experiencia de emigración en la construcción de la identidad cultural. Los actos de identificación de los no emigrantes suelen usar un lenguaje generalizado referido a un universal de casos para construir la identidad cultural andaluza, generalizaciones que describen características de Andalucía o de los andaluces. Los emigrantes, sin embargo, suelen usar su experiencia personal de emigración como recurso para construir sus actos 
de identificación con Andalucía. Su discurso está compuesto por narraciones particulares y situadas, con un agente concreto que, por lo general, es el mismo hablante o alguna persona cercana. Para ellos, la comparación con el otro es un recurso casi ineludible, que es tratado con una gran carga emocional que idealiza la "tierra perdida" y la extraña.

Esta influencia de la experiencia de emigración en la construcción de la identidad andaluza debe ser matizada y estudiada más a fondo en posteriores estudios. La identidad cultural resulta de la toma de consciencia de los grupos humanos en situaciones de contraste y confrontación con otros grupos. El desarrollo de una identidad comunal representa una toma de posición frente a la presencia de un agente externo, que representa un "potencial peligro" para asimilar en el terreno simbólico del propio grupo. En las posiciones teóricas presentadas, se tiende a explicar la identidad cultural y sus manifestaciones de autodefensa o ataque en términos de la consciencia de la diferencia y del contraste en situaciones de interacción social, sirviendo los agentes externos como catalizadores para la manifestación de la identidad cultural. Dichos desarrollos remiten de nuevo a la idea del carácter situado y funcional de la identidad cultural y reclaman el uso de perspectivas analíticas que consideren al alter, al otro, como frontera de uno mismo para entender los propios procesos de construcción identitaria.

\section{Referencias}

Bakhtin, M. (1986). Speech genres and other late essays. Austin: University of Texas Press.

Barth, F. (1976). Los grupos étnicos y sus fronteras. México D.F.: Fondo de Cultura Económica.

Bruner, J. S. (1991). Self-making and world-making. Journal of Aesthetic Education, 25(1), 67-78.

Clegg, J. W. \& Salgado, J. (2011). From Bakhtinian theory to a dialogical psychology. Culture \& Psychology, 17(4), 520-533. DOI: 10.1177/1354067X11418546
Espinosa, A., Soares-da Silva A., Contreras, C., Cueto, R., García A., Ortolano, F., Valencia, J. \& Vera, A. (2017). Identidad nacional y sus relaciones con la ideología y el bienestar en cinco países de América Latina. Avances en Psicología Latinoamericana, 35(2), 363-386.

Esteban-Guitart, M. (2008). ¿Por qué nos importa tanto el tema de la identidad? Aposta: Revista de Ciencias Sociales, 39, 1-15.

Esteban-Guitart, M. \& Vila, I. (2015). The voices of newcomers. A qualitative analysis of the construction of transnational identity. Psychosocial Intervention, 24(1), 17-25.

Guerrero, A. \& Cuadra, J. A. (2013). Construcción psicosocial de la alteridad: racismo en México. Cultura y Representaciones Sociales, 8(15), 74-96.

Hall, S. (1990). Cultural identity and diaspora. En J. Rutherford (ed.), Identity: community, culture, difference (pp. 222-237). Londres: Lawrence and Wishart.

Jenkins, R. (1997). Rethinking ethnicity: arguments and explorations. Londres: Sage.

Labrador, J. (2001). Identidad e inmigración. Un estudio cualitativo con emigrantes peruanos en Madrid. Madrid: Servicio de Publicaciones de Universidad Pontificia de Comillas.

Macías-Gómez-Estern, B. (2013). “And now I am here..., but then we were there": space and social positioning in Andalusian migrant's narratives. Journal of Multicultural Discourses, 8(3), 195-212.

Macías-Gómez-Estern, B., García, J. \& Sánchez, J. A. (2008). Cultural identity and immigration. En B. Van Oers, E. Elbers, R. Van der Veer \& W. Wardekker (eds.), The transformation of learning. Perspectives from activity theory (pp. 201-218). Cambridge: Cambridge University Press.

Macías-Gómez-Estern, B., Amián, J. G., Sánchez, J. A. \& Marco, M. J. (2010). Literacy and the formation of cultural identity. Theory and Psychology, 20(2), 231-250. 
Macías-Gómez-Estern, B. \& De la Mata Benítez, M. L. (2013). Narratives of migration: Emotions and the interweaving of personal and cultural identity through narrative. Culture \& Psychology, 19(3), 348-368.

Mead, G. H. (1974 [1934]). Mind, self \& society. From the standpoint of a social behaviorist. Chicago: The University of Chicago Press.

Montero, M. (1984). Ideología, alienación e identidad nacional. Una aproximación psicosocial al ser venezolano. Caracas: Universidad Central de Venezuela.

Moreno, I. (1993). Andalucía: identidad y cultura (estudios de antropología andaluza). Ágora: Málaga

Pujadas, J. J. (1993). Etnicidad. Identidad cultural de los pueblos. Madrid: Eudema.

Sakki, I. \& Pettersson, K. (2016). Discursive constructions of otherness in populist radical right political blogs. European Journal of Social Psychology, 46(2), 156-170.

Shweder, R. A. (1990). Cultural psychology - What is it? En J. W. Stigler, R. A. Shweder, \& H.
Gilbert (eds.), Cultural psychology: essays of comparative human development (pp. 1-43). Nueva York: Cambridge University Press.

Smith, A. D. (1991). National identity. Reno: University of Nevada Press.

Tajfel, H. (1984). Grupos humanos y categorías sociales. Barcelona: Herder.

Turner, J. C. (1991). Social influence. Milton Keynes: Open University Press.

Vallejos, J., Wigstrom, E., Olivares, T., Yzerbyt, V. \& Estrada, C. (2015). Variables asociadas a la identidad social satisfactoria y sus efectos diferenciales en el esencialismo endo y exogrupal. Avances en Psicología Latinoamericana, 33(2), 175-188.

Vygotski, L. S. (1993). Pensamiento y lenguaje. Obras escogidas II. Madrid: Aprendizaje Visor. Yuval-Davis, N. (2010). Theorizing identity: beyond the 'us' and 'them' dichotomy. $P a$ tterns of Prejudice, 44(3), 261-280. DOI: $10.1080 / 0031322 X .2010 .489736$ 
